\title{
A STUDY ON TONSIL CORE CULTURE IN CHRONIC AND RECURRENT TONSILLITIS
}

\author{
Ali Shaik1, Thota Gopi Krishna², Asha Parveen Sayyad ${ }^{3}$ \\ ${ }_{1}^{1}$ Assistant Professor, Department of ENT, KIMS and RF, Amalapuram, Andhra Pradesh, India. \\ 2Deputy Medical Superintendent, SSCL, Bhupalapally, Andhra Pradesh, India. \\ ${ }_{3}^{3}$ Assistant Professor, Department of Community Medicine, KIMS and RF, Amalapuram, Andhra Pradesh, India.
}

ABSTRACT

\section{BACKGROUND}

Palatine tonsils are the components of Mucosa Associated Lymphoid Tissue (MALT) of pharynx. Embryologically, they develop from second branchial arch and play an important role in immune system. These are lined by stratified squamous epithelium in continuation with pharyngeal mucosa. The surface epithelium of tonsil will invaginate to form crypts. These crypts contain cheesy material which constitute food debris, keratin and bacteria. Infection of tonsil, tonsillitis was a more common infection of humans. During tonsillitis, the bacteria inhabiting the crypts will multiply and secrete toxins. This inflammation of tonsil tissue will lead to WBC infiltration, finally leading to swollen and congested tonsils. Antibiotics play an important role in treatment of acute tonsillitis. Appropriate antibiotic selection from tonsil surface swab culture for an adequate duration will prevent the continuation and spread of infection. Contrary to this surface swab culture oriented antibiotic selection, few studies reported that the bacteria causing tonsillitis may also be present in deep tonsillar tissues. Hence, the organisms grown from tonsillar surface swab cultures may not be representative of actual infective organism always.

\section{MATERIALS AND METHODS}

This descriptive study was carried out as a prospective study among 60 patients who underwent tonsillectomy at a tertiary care hospital. Data was collected about various socio-demographic factors, clinical information and details about routine lab examinations. After tonsillectomy, the specimen was transferred in sterile water for aerobic culture and KOH mount.

\section{RESULTS}

Out of 33 positive cultures, S. aureus (48.5\%) was grown majorly followed by H. influenzae (18.2\%), S. pyogenes (12.1\%), Pseudomonas (9.1\%), K. pneumoniae (6.1\%), S. pneumoniae (3\%) and no fungal elements were detected.

\section{CONCLUSION}

Surface cultured micro-organism represent more of normal commensals, which is a false indicator of the pathogen in the core. The findings of this study are in support of directing rational therapy against tonsillar core pathogens like Staph. aureus, H. influenza, Pseudomonas, Klebsiella with antibiotics like Gentamycin, Amikacin and Ciprofloxacin.

\section{KEY WORDS}

Tonsillitis, Acute Tonsillitis, Chronic Tonsillitis, Core Culture of Tonsils, Antibiotic Resistance, Tonsillectomy.

HOW TO CITE THIS ARTICLE: Shaik A, Krishna TG, Sayyad AP. A study on tonsil core culture in chronic and recurrent tonsillitis. J. Evolution Med. Dent. Sci. 2018;7(29):3308-3311, DOI: 10.14260/jemds/2018/745

\section{BACKGROUND}

Immune system in the body comprised of many components, of them palatine tonsils of Waldeyer ring has an important role. Palatine tonsils are paired masses of lymphoid tissue present in lateral wall of oropharynx. They are bounded anteriorly by palatoglossal arches and posteriorly by palatopharyngeal arches. These are mucosal associated subepithelial tissue present at the entrance of oropharynx. Embryologically, they develop from second pharyngeal pouch. Immunological activity of tonsils is more during first decade of life. ${ }^{1}$ The surface epithelium of tonsil is invaginated to form tonsillar crypts.

'Financial or Other Competing Interest': None.

Submission 07-06-2018, Peer Review 30-06-2018,

Acceptance 07-07-2018, Published 16-07-2018.

Corresponding Author:

Asha Parveen Sayyad,

Department of Community Medicine,

KIMS and RF, Amalapuram-533201,

East Godavari District,

Andhra Pradesh, India.

E-mail:dr.alishaik@gmail.com

DOI: $10.14260 /$ jemds $/ 2018 / 745$

\section{(c) $(\mathbf{P})$}

The number of tonsillar crypts may vary from 15 to 20 . They appear as multiple opening on the medial surface of tonsil. One of the crypts is larger and it is called as crypta magna. These tonsillar crypts may also give rise to secondary crypts.

Tonsils play an important role in inducing secretory immunity and regulation of immunoglobulin production. Tonsils will produce local antibodies and B lymphocytes. These will migrate to other sites of pharynx and periglandular lymphoid tissue where they produce antibodies. T. lymphocytes present in tonsils will also produce gamma interferons and other lymphokines. But the exact role of tonsillar $\mathrm{T}$ cells in tumour response is not known. Tonsils are immunologically active between 4 and 10 years of age. After puberty, regression of tonsillar tissue will take place. This results in decrease in B cell population and relative increase in $\mathrm{T}$ cell to $\mathrm{B}$ cell ratio. However, immunoglobulin function is not much affected. Even in the tonsillar tissues of individuals in $8^{\text {th }}$ decade, considerable $\mathrm{B}$ lymphocyte function is seen, provided if they are clinically healthy. Tonsils are the places for proliferation of $\mathrm{T}$ and $\mathrm{B}$ lymphocytes as a part of their immunological function. Tonsils will participate in selection of relevant clones of $\mathrm{T}$ and $\mathrm{B}$ lymphocytes in relation to the microorganisms of 
oropharynx. To start this action, the antigens at pharyngeal surface will cross the surface epithelium of tonsil and are presented to the local immune system by antigen representing cells. During acute infection of tonsils, bacteria invading the crypts may also enter tonsil core tissue. These bacteria will secrete toxins and other products which in turn cause swelling, necrosis and sometimes superficial ulceration also. Bacteria that invaded the tonsil core tissue will remain there and act as nidus for further infections. This condition will lead to recurrent infection of tonsil tissue.

As the child becomes older, the tonsillar tissue decrease in size and sub-epithelial tissue becomes fibrotic and crypts are filled with cheesy material. This cheesy material constitutes keratin, food debris and bacteria. During tonsillitis the bacteria inhabiting the crypts will multiply and secrete toxins. This inflammation of tonsil tissue will lead to WBC infiltration finally leading to swollen and congested tonsils. Sometimes ulcers, membrane and necrosis of tonsillar tissue can occur. ${ }^{2}$ Sometimes, these chronically infected tonsils will enlarge. This enlargement is due to increase in amount of connective tissue, presence of lymphocytes and neutrophils in large number. Occasionally, in chronically enlarged tonsils, keratin cysts and chronic abscesses have been reported. Whether it is acute or chronic infection of tonsil, it is often associated with proliferation of microorganisms from tonsillar core parenchyma.

Antibiotics play an important role in treatment for acute tonsillitis. Appropriate antibiotic selection for an adequate duration will prevent the continuation and spread of infection. Insufficiencies in the treatment strategy can lead to continuation of disease process and sometimes relocation of infection on tonsil surface. 3 For appropriate antibiotic selection, one can adopt swab cultures from tonsil surface. These culture and sensitivity reports will help not only in the selection of appropriate culture-guided antibiotic selection, but also for prophylaxis in carriers. In contrary to this surface swab culture oriented antibiotic selection, few studies reported that the bacteria causing tonsillitis may also present in deep tonsillar tissues. ${ }^{4}$ Hence, the organisms grown from tonsillar surface swab cultures may not be representative of actual infective organism always. ${ }^{5}$

So identification and isolation of bacteria from the core of tonsil tissue is important for many reasons. These cultured bacteria from tonsillar core tissue may be responsible for all the changes described. Most of the times the treatment for tonsillar infection is dependent on the results of surface swab cultured microbes. As these microbes may not be representative of actual infective aetiology, hence selection of antibiotics based on these cultures will lead to failure of treatment and thereby failure in eradication of tonsillar core microbes. This inappropriate selection of antibiotics and their insufficient penetration into tonsillar core tissue will cause persistence or relocation of infection. Lastly, the harboured bacteria in the core of tonsil may exert a protective effect on other pathogens. This protective effect may lead to development of resistance against antibiotics. Medical management with antibiotics and anti-inflammatory agents is sufficient for the treatment of acute tonsillitis. But in cases of recurrent and chronic tonsillitis, tonsillectomy is the treatment of choice. 6

\section{Aims and Objectives}

1. To obtain core culture of the tonsils in patients presenting with recurrent/ chronic tonsillitis.

2. To see for presence of any fungal elements in core tissue of tonsil.

\section{MATERIALS AND METHODS}

This descriptive study was carried out as a prospective study among 60 patients of various age groups (Table 1) who underwent surgery for recurrent tonsillitis in the Department of ENT at a tertiary care facility excluding HIV/ immunecompromised patients, patients on antibiotics within the past 3 weeks and patients undergoing tonsillectomy for reasons other than recurrent/ chronic tonsillitis. Prior to surgery data was collected about various socio-demographic factors, clinical information, physical examination and details about routine lab examinations. After doing tonsillectomy by dissection and snare method, the tissue was taken from the core of the tonsil under aseptic conditions and excised tissue was kept in sterile water and sent for culture. For culture freshly prepared blood agar with neomycin, yeast extract, hemin and vitamin $\mathrm{K}$ is adequate. Plates are incubated at $37^{\circ} \mathrm{C}$ on anaerobic jar with $10 \% \mathrm{CO}_{2}$. The sample was cultured in aerobic media for a period of 24 - 48 hours.

\section{RESULTS}

Out of 60 cultures done in aerobic media, 33 cultures are positive. The most common organisms cultured from the core in patients with recurrent/ chronic tonsillitis were S. aureus $(48.5 \%)$, H. influenzae $(18.2 \%)$, S. pyogenes $(12.1 \%)$, Pseudomonas (9.1\%), K. pneumoniae (6.1\%), S. pneumoniae (3\%) with one sample being contaminated $(3 \%)$ and no fungal element detected by $\mathrm{KOH}$ mount (Table 2).

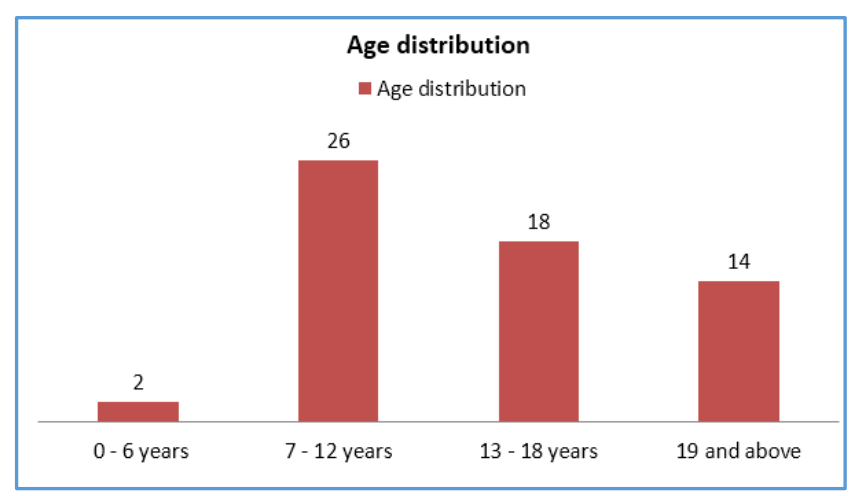

Table 1

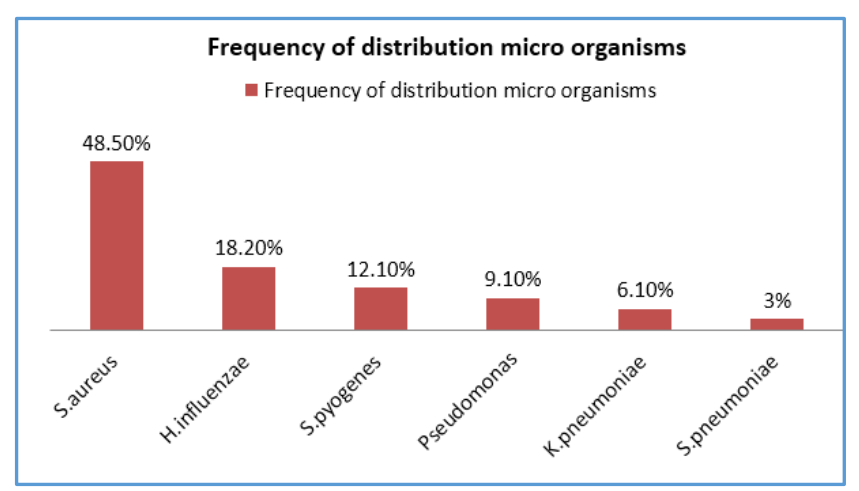

Table 2 


\section{DISCUSSION}

The microbial population of the oral cavity is complex. It is a mixture of many aerobic bacteria and obligate anaerobes. The differences in the quantitative existence of the anaerobic microflora may be due to difference in the oxygen concentration in parts of the oral cavity. Aerobic and anaerobic bacteria will exist at 1: 10 ratio in saliva approximately. Viral infections can cause changes in the bacterial flora of oral cavity. These changes may be attributed to the increased adherence of Staph. aureus as well as gram-negative enteric organisms. During illness-free periods, the colonisation of gram-negative enteric organisms and Staph. aureus was found to vary. The oropharyngeal colonisation of gram-negative enterics was found to vary from 12 to 18 percent for and from 5 to 14 percent for Staph. aureus. During an episode of viral URTI, the colonisation of gram-negative enterics and Staph. aureus increased to 60 percent and 43 percent, respectively. However, these changes were transient and might be a factor contributing to increased risk of serious bacterial infection.

Interference of these bacteria between various microorganisms may be responsible for the maintenance of normal flora in oropharynx. This normal flora will help an individual in maintaining an efficient resistance barrier against many pathogens. However, the equilibrium between these organisms can be disrupted by antimicrobial therapy. Usage of ampicillin, cephalothin, tetracycline and chloramphenicol have been shown to suppress alpha haemolytic streptococci and promote the emergence of gram-negative enteric organisms. Antibiotic like penicillin can enhance the acquisition of $\beta$-lactamase producing bacteria in the oropharynx. These will lead to shift in the percentage of normal microbial flora and thereby emergence antibiotic resistant microbes. Finally, this will lead to failure of drug therapy in the management of oropharyngeal infections, especially tonsillitis.

Over the past 30 years, several studies have been done to look for or detect the cause of recurrent/ chronic tonsillitis. The management of these conditions has changed over the last 3 decades due to the results obtained by these studies. As a norm all cases that presented with acute/recurrent/chronic tonsillitis, a throat swab was the first line of management.

Normal bacterial flora present in the oral cavity and oropharynx were cultured in most cases and antibiotic therapy was instituted based on the results obtained from the cultures grown by the throat swab. Several studies have disapproved this theory. Studies done by different authors have stated that pathogens found in the tonsillar surface vary from those found in the tonsillar core and antibiotic therapy should be targeted towards the pathogens that are found in the core in cases of recurrent/ chronic tonsillitis.3,4,5,6,7,8,9

The most common organism isolated in our study from tonsillar core is Staphylococcus aureus (48.5\%) followed by H. influenzae (18.2\%). There were no fungal elements identified in our study. In Abhay Kumar et al,10 it was Staphylococcus aureus followed by GABHS. In Babaiwa UF et al,11 the most common organism isolated was Staphylococcus aureus (69\%) followed by Streptococcus pneumoniae (14\%). In Abdelazeem ME et al,12 the common organism cultured was Staph. aureus (77.7\%) followed by GABHS (18.5\%). In Mustafa Gul et al, most common organism was GABHS followed by Staphylococcus aureus. In Salman Mutiullah Shaikh et al,13 the most common organism was Streptococcus pneumoniae (86.56\%) followed by Staphylococcus aureus $(47.76 \%)$. Bista $\mathrm{M}$ et $\mathrm{al}^{9}$ showed Streptococcus viridans and Streptococcus pneumoniae and in Agarwal A et al14 Streptococcus viridans and Branhamella catarrhalis (71.13\%). So majority of tonsil core culture studies identified Staph. aureus as the most common pathogen.

\section{CONCLUSION}

Numerous studies have been done in comparing the surface to the core of the tonsils in patients presenting with recurrent and chronic tonsillitis. These studies have proved that surface swab cultures of the tonsils showed normal bacterial flora present in the oral cavity and oropharynx, hence taking a surface swab culture is said to be a false indicator of the pathogen in the core and hence unreliable.

Organisms isolated from the core in cases of recurrent/chronic tonsillitis proved to be different from those isolated from the surface swab cultures; therefore selection of an appropriate antibiotic from surface cultures is of little or no significance. In this study, it was found that in recurrent/chronic tonsillitis, the most common organisms were Staph. aureus, H. influenzae, GABHS and Pseudomonas. The findings of this study are in support of directing rational therapy against tonsillar core pathogens like Staph. aureus, H. influenza, Pseudomonas, Klebsiella with antibiotics like Gentamycin, Amikacin and Ciprofloxacin.

\section{REFERENCES}

[1] Uppal K, Bais AS. Tonsillar microflora--superficial surface vs deep. J Laryngol Otol 1989;103(2):175-7.

[2] Discolo CM, Darrow DH, Koltai PJ. Infectious indications for tonsillectomy. Pediatr Clin North America 2003;50(2):445-58.

[3] Gul M, Okur E, Ciragil P, et al. The comparison of tonsillar surface and core cultures in recurrent tonsillitis. American Journal of Otolaryngology 2007;28(3):173-6.

[4] Mahakit P, Moungthong G, Sombulna T, et al. The correlation of micro-organisms between tonsillar crypt culture and tonsillar core culture in chronic tonsillitis. J Med Assoc Thai 2005;88(Suppl 3):S82-8.

[5] Syrylo A, Wojdas A, Jurkiewicz D. Bacterial flora of the tonsillar surface versus tonsillar core in chronic tonsillitis. Otolaryngol Pol 2007;61(4):598-601.

[6] Brodsky L, Nagy M, Volk M. The relationship of tonsil bacterial concentration to surface and core cultures in chronic tonsillar disease in children. Int J Pediatr Otorhinolaryngol 1991;21(1):33-9.

[7] Kurien M, Stanis A, Job A, et al. Throat swab in chronic tonsillitis: How reliable and valid is it? Singapore Med J 2000;41(7):324-6.

[8] Ahmad I, Anwar MS, Naeem K, et al. Surface vs. core tonsillar aerobic and anaerobic flora in recurrent tonsillitis. Pak Paed J 1998;22(4):185-8.

[9] Bista M, Sinha BK, Amatya RCM, et al. Comparison of core and surface cultures in recurrent tonsillitis. Journal of Institute of Medicine 2005;27(3):60-5. 
[10] Kumar A, Gupta V, Chandra K, et al. Clinico bacteriological evaluation of surface and core microflora in chronic tonsillitis. Indian J Otolaryngol Head Neck Surg 2005;57(2):118-20.

[11] Babaiwa UF, Onyeagwara NC, Akerele JO. Bacterial tonsillar microbiota and antibiogram in recurrent tonsillitis. Biomedical Research 2013;24(3):298-302.

[12] Elsherif AM, Abdelrahman YOP, Abdelazeem MH. Discrepancy between Surface and Core culture in children with chronic tonsillitis and incidence of post tonsillectomy bacteraemia. Al-Azhar Assiut Medical J 2011;9(1):171-82.
[13] Shaikh SM, Afzal JM, Naila TM, et al. Bacteriology of tonsilar surface and core in patients with recurrent tonsillitis, undergoing tonsillectomy. Medical Channel 2009;16(4):95-7.

[14] Agrawal A, Kumar D, Goyal A, et al. Bacteriological evaluation and their antibiotic sensitivity pattern in tonsillitis. IOSR-J Den Med Sci 2014;13(3):51-5. 